\title{
Erratum to: Exploring the effects of coexisting amyloid in subcortical vascular cognitive impairment
}

\author{
Elizabeth Dao ${ }^{1}$, Ging-Yuek Robin Hsiung ${ }^{2}$, Vesna Sossi ${ }^{3}$, Claudia Jacova ${ }^{4}$, Roger Tam ${ }^{5,6}$, Katie Dinelle ${ }^{7}$,
} John R. Best ${ }^{1}$ and Teresa Liu-Ambrose ${ }^{1,8^{*}}$

\section{Erratum}

After publication of the original article [1], it came to the authors' attention that there were data entry errors in the following variables: age, sex, education, MOCA, WML volume, and ADAS-Cog. Changes have been made to Tables 1, 2, 3 and 4, to reflect the updated results, which are published in their correct version here. However, the authors note that the study conclusions have not changed.

\begin{abstract}
Author details
'Djavad Mowafaghian Centre for Brain Health, University of British Columbia, 2215 Wesbrook Mall, Vancouver, BC V6S 0A9, Canada. ${ }^{2}$ Department of Medicine, University of British Columbia, UBC Hospital S152, 2211 Wesbrook Mall, Vancouver, BC V6T 2B5, Canada. ${ }^{3}$ Department of Physics and

Astronomy, University of British Columbia, 6224 Agricultural Road, Vancouver, BC V6T 1Z1, Canada. ${ }^{4}$ School of Professional Psychology, Pacific University, 190 SE 8th Avenue, Hillsboro, OR 97123, USA. ${ }^{5}$ Department of Radiology, University of British Columbia, 3350-950W 10th Avenue, Vancouver, BC V5Z 1M9, Canada. ${ }^{6} \mathrm{MS} / \mathrm{MRI}$ Research Group, University of British Columbia, 2215 Wesbrook Mall, Vancouver, BC V6S OA9, Canada. ${ }^{7}$ PET Group, Vancouver Hospital and Health Science Centre, Brain Research Centre, 2211 Westboork Mall, Vancouver, BC V6T 2B5, Canada. ${ }^{8}$ Department of Physical Therapy, University of British Columbia, 212-2177 Wesbrook Mall, Vancouver, BC V6T 1Z3, Canada.
\end{abstract}

Published online: 31 August 2016

\section{Reference}

1. Dao E, Hsiung GY, Sossi V, Jacova C, Tam R, Dinelle K, et al. Exploring the effects of coexisting amyloid in subcortical vascular cognitive impairment. BMC Neurol. 2015;15:197. doi:10.1186/s12883-015-0459-1.

\footnotetext{
* Correspondence: teresa.ambrose@ubc.ca

'Djavad Mowafaghian Centre for Brain Health, University of British Columbia, 2215 Wesbrook Mall, Vancouver, BC V6S 0A9, Canada

${ }^{8}$ Department of Physical Therapy, University of British Columbia, 212-2177

Wesbrook Mall, Vancouver, BC V6T 1Z3, Canada
} 
Table 1 Descriptive characteristic

\begin{tabular}{lcc}
\hline Variable & Mean & SD \\
\hline Age & 71.73 & 7.91 \\
Female Sex, No. (\%) & 7 & 32 \\
Education, No. (\%) & & \\
$\quad$ High school education & 6 & 27 \\
$\quad$ Trade or professional certificate or diploma & 2 & 9 \\
$\quad$ University education & 14 & 64 \\
MMSE (max. score 30) & 27.50 & 1.95 \\
MOCA (max. score 30) & 23.32 & 2.08 \\
WHR & 0.91 & 0.08 \\
BMI & 26.95 & 4.78 \\
PIB-positive, No. (\%) & 6 & 27.27 \\
Global PIB BP ND & 0.07 & 0.23 \\
WML volume (mm ${ }^{3}$ ), $n=16$ & 2004.40 & 2761.15 \\
Cognitive Assessments & & \\
ADAS-Cog (max. score 70) & 9.63 & 3.88 \\
Exit-25 (max. score 50) & 10.59 & 4.38 \\
Stroop CW-C, sec. & 61.44 & 26.01 \\
Trails B-A, sec. & 50.51 & 24.84 \\
Digits F-B, sec. & 3.23 & 2.79 \\
\hline SD Standad Devion, MMSE Mni-Mental State Examing & & \\
\hline
\end{tabular}

SD Standard Deviation, MMSE Mini-Mental State Examination, MOCA Montreal Cognitive Assessment; WHR Waist-to-Hip Ratio, BMI Body Mass Index, ADAS-Cog Alzheimer's Disease Assessment Scale - Cognitive subscale, Exit-25 Executive Interview Test, Stroop CW-W Stroop Color Words minus Stroop colored x's, Trails $B-A$ Trails $B$ (numbers and letters) minus Trails A (numbers), Digits F-B Digits Forwards minus Digits Backwards

Table 2 Correlation matrix

\begin{tabular}{|c|c|c|c|c|c|c|c|c|c|c|}
\hline & $\mathrm{PIB} B P_{\mathrm{ND}}$ & Age & Education & APOE ع4 & ADAS-Cog & MOCA & EXIT 25 & Digits & Stroop & Trails \\
\hline \multicolumn{11}{|l|}{$\mathrm{PIB} B P_{\mathrm{ND}}$} \\
\hline Age & -0.00 & & & & & & & & & \\
\hline Education & -0.23 & -0.39 & & & & & & & & \\
\hline APOE ع4 & 0.36 & 0.09 & -0.20 & & & & & & & \\
\hline ADAS-Cog & $0.53^{*}$ & 0.16 & -0.11 & $0.53^{*}$ & & & & & & \\
\hline MOCA & $-0.54^{*}$ & -0.20 & 0.06 & -0.11 & $-0.45^{*}$ & & & & & \\
\hline EXIT-25 & 0.13 & 0.20 & -0.20 & 0.25 & $0.49^{*}$ & $-0.52^{*}$ & & & & \\
\hline Digits & 0.02 & 0.17 & -0.35 & $-0.45^{*}$ & -0.30 & 0.13 & -0.04 & & & \\
\hline Stroop & 0.17 & -0.06 & 0.02 & -0.11 & 0.27 & -0.29 & 0.34 & 0.04 & & \\
\hline Trails & 0.19 & -0.08 & -0.25 & $0.48^{*}$ & 0.15 & -0.18 & 0.29 & 0.01 & 0.13 & \\
\hline
\end{tabular}

*significant at $p \leq 0.05$ 
Table 3 Multiple linear regression models assessing the contribution of PIB retention on ADAS-Cog

\begin{tabular}{|c|c|c|c|c|c|c|}
\hline Independent variables & $R^{2}$ & Adjusted $R^{2}$ & $\mathrm{R}^{2}$ Change & Unstandardized B (Standard Error) & Standardized $\beta$ & $P$-Value \\
\hline \multicolumn{7}{|l|}{ Model 1} \\
\hline $\mathrm{PIB} \mathrm{BP}_{\mathrm{ND}}$ & 0.28 & 0.24 & - & $9.09(3.29)$ & 0.53 & 0.01 \\
\hline \multicolumn{7}{|l|}{ Model 2} \\
\hline Step 1 & 0.30 & 0.18 & 0.30 & & & \\
\hline Age & & & & $0.07(0.11)$ & 0.14 & 0.54 \\
\hline Education & & & & $0.41(1.86)$ & 0.05 & 0.83 \\
\hline APOE $\varepsilon 4$ & & & & $4.30(1.64)$ & 0.53 & 0.02 \\
\hline Step 2 & 0.44 & 0.31 & $0.15^{*}$ & & & \\
\hline Age & & & & $0.09(0.10)$ & 0.18 & 0.37 \\
\hline Education & & & & $1.15(1.74)$ & 0.14 & 0.52 \\
\hline APOE $\varepsilon 4$ & & & & $3.20(1.59)$ & 0.39 & 0.06 \\
\hline $\mathrm{PIB}_{\mathrm{BP}} \mathrm{ND}_{\mathrm{N}}$ & & & & $7.23(3.42)$ & 0.42 & 0.05 \\
\hline
\end{tabular}

*significant at $p \leq 0.05$

Table 4 Multiple linear regression models assessing the contribution of PIB retention on MOCA

\begin{tabular}{|c|c|c|c|c|c|c|}
\hline Independent variables & $R^{2}$ & Adjusted $R^{2}$ & $R^{2}$ Change & Unstandardized B (Standard Error) & Standardized $\beta$ & $P$ - Value \\
\hline \multicolumn{7}{|l|}{ Model 1} \\
\hline$P I B B P_{N D}$ & 0.29 & 0.26 & - & $-5.00(1.74)$ & -0.54 & 0.01 \\
\hline \multicolumn{7}{|l|}{ Model 2} \\
\hline Step 1 & 0.05 & -0.11 & 0.05 & & & \\
\hline Age & & & & $-0.06(0.07)$ & -0.21 & 0.41 \\
\hline Education & & & & $-0.22(1.16)$ & -0.05 & 0.85 \\
\hline APOE $\varepsilon 4$ & & & & $-0.43(1.02)$ & -0.10 & 0.68 \\
\hline Step 2 & 0.37 & 0.22 & $0.32^{*}$ & & & \\
\hline Age & & & & $-0.07(0.06)$ & -0.28 & 0.20 \\
\hline Education & & & & $-0.80(0.99)$ & -0.18 & 0.43 \\
\hline APOE $\varepsilon 4$ & & & & $0.44(0.91)$ & 0.10 & 0.63 \\
\hline$P I B B_{N D}$ & & & & $-5.71(1.95)$ & -0.62 & 0.01 \\
\hline
\end{tabular}

